\title{
THE CONFIGURATION SPACE OF GAUGE THEORY ON OPEN MANIFOLDS OF BOUNDED GEOMETRY
}

\author{
J ÜRGEN EICHHORN \\ Fachbereich Mathematik, Universität Greifswald \\ Jahnstraße 15a, 17487 Greifswald, Germany \\ E-mail: eichhorn@math-inf.uni-greifswald.d400.de \\ GERD HEBER \\ GMD-FIRST \\ Rudower Chaussee 5, Geb. 13.10, 12489 Berlin, Germany \\ E-mail: heber@first.gmd.de
}

\begin{abstract}
We define suitable Sobolev topologies on the space $\mathcal{C}_{P}\left(B_{k}, f\right)$ of connections of bounded geometry and finite Yang-Mills action and the gauge group and show that the corresponding configuration space is a stratified space. The underlying open manifold is assumed to have bounded geometry.

1. Introduction. Consider a differential equation $A u=0$ (D). If $\mathcal{S}$ is the set of all possible solutions and $\mathcal{G}$ the automorphism group of (D) then the configuration space $\mathcal{S} / \mathcal{G}$ plays a decisive role for the solution theory of (D). At the first instance, $\mathcal{S} / \mathcal{G}$ is a senseless object until there are introduced suitable topologies and performed completions. In this paper, we present a canonical approach for gauge theory on open manifolds, i.e. for the equation $\delta^{\omega} R^{\omega}=0$. For compact manifolds, there exists a nice and complete representation given by Kondracki et al. in [14], [15]. Unfortunately all of the arguments become completely wrong on open manifolds, e.g. the properness of the action of the completed diffeomorphism group on the space of Riemannian metrics, mapping properties of elliptic operators and many other features of elliptic theory. In this paper, we restrict ourselves to the case of bounded geometry and give a complete topological description of the configuration space of gauge theory.
\end{abstract}

1991 Mathematics Subject Classification: Primary 81T13; Secondary 58A35,58E15.

Research of the second author supported by the Real World Computing Partnership, Japan.

For the second author this was a preliminary study for the application of the PROMOTER programming model in lattice gauge theory.

The paper is in final form and no version of it will be published elsewhere. 
Let $\left(M^{n}, g\right)$ be an open Riemannian manifold satisfying the following two conditions $(I)$ and $\left(B_{k}\right)$,

$$
r_{\mathrm{inj}}(M)=\inf _{x \in M} r_{\mathrm{inj}}(x)>0
$$

$$
\left|\left(\nabla^{g}\right)^{i} R^{g}\right| \leq C_{i}, \quad 0 \leq i \leq k, k>n / 2+1 .
$$

Here $r_{\text {inj }}$ denotes the injectivity radius. $r_{\text {inj }}>0$ implies the completeness of the metric. See [6] for a proof.

We motivate $(I)$ and $\left(B_{k}\right)$ as follows. Compact Riemannian manifolds always satisfy $(I)$ and $\left(B_{k}\right)$ for all $k$. Hence $(I)$ and $\left(B_{k}\right)$ mean that the geometry at infinity is bounded in a certain sense which seems physically reasonable. A second reason for assuming $(I)$ and $\left(B_{k}\right)$ is more technical. $(I),\left(B_{k}\right), k \geq r>\frac{n}{2}+1$ assure several important and in the sequel needed properties of Sobolev spaces as the embedding theorem 2.2 and the module structure theorem 2.3. Our general idea constructing completed nonlinear spaces is starting with bounded objects (maps, connections) and completing by adding Sobolev perturbations.

Let $P(M, G) \rightarrow M$ be a $G$-principal fibre bundle, $G$ compact with Lie algebra $\mathbf{g}$, $\mathbf{g}_{P}=P \times_{\text {ad }} G, \mathcal{C}_{P}\left(B_{k}\right.$, fin) the set of $G$-connections satisfying

$$
\left(B_{k}(\omega)\right) \quad\left|\left(\nabla^{\omega}\right)^{i} R^{\omega}\right| \leq D_{i}, 0 \leq i \leq k,
$$

and

$$
\mathcal{Y} \mathcal{M}(\omega):=\frac{1}{2} \int_{M}\left|R^{\omega}\right|_{x}^{2} d \operatorname{vol}_{x}(g)<\infty
$$

and let

$$
\mathcal{G}=\left\{\varphi: P \stackrel{\simeq}{\longrightarrow} P \mid \varphi \text { covers id }_{M}, \varphi(p \cdot g)=\varphi(p) \cdot g, \sum_{i=0}^{k-1} \sup _{p \in P}\left|\nabla^{i} d \varphi\right|_{p}<\infty\right\},
$$

where $P$ is endowed in a canonical manner with a metric of bounded geometry. We introduce in $\mathcal{C}_{P}\left(B_{k}\right.$, fin $), \mathcal{G}$ Sobolev topologies, obtain $\mathcal{C}_{P}^{r}\left(B_{k}\right.$, fin $), k \geq r>n / 2+1$, where $\mathcal{C}_{P}^{r}\left(B_{k}\right.$, fin) splits into a topological sum of its components,

$$
\mathcal{C}_{P}^{r}\left(B_{k}, \text { fin }\right)=\sum_{i \in I} \operatorname{comp}\left(\omega_{i}\right)=\sum_{i \in I} \omega_{i}+\Omega^{1, r}\left(\mathbf{g}_{P}, \nabla^{\omega}\right) .
$$

$\mathcal{G}^{r+1}=\mathcal{G}^{r+1}\left(\operatorname{comp}\left(\omega_{i}\right)\right)$ is adapted to $\operatorname{comp}\left(\omega_{i}\right)$, is a Hilbert-Lie group and acts smoothly on $\operatorname{comp}\left(\omega_{i}\right)$.

Our main task is to study

$$
\operatorname{comp}\left(\omega_{i}\right) / \mathcal{G}^{r+1}\left(\operatorname{comp}\left(\omega_{i}\right)\right) .
$$

We show that this is a stratified space with in general uncountable number of strata which are labelled by conjugacy classes of isotropy groups. Here we assume additionally a certain spectral condition.

The paper is organized as follows. In Section 2 we recall some facts on the space $\mathcal{C}_{P}^{r}\left(B_{k}\right.$, fin) which are contained in [4]. Section 3 is devoted to the detailed definition of the completed gauge group $\mathcal{G}^{r+1}\left(\omega_{0}\right)$. We show in Section 4 that $\mathcal{G}^{r+1}\left(\omega_{0}\right)$ acts smoothly on 
$\operatorname{comp}\left(\omega_{0}\right)$, the orbits are submanifolds and the action admits a slice. Section 5 , finally, contains our main theorem, the stratification of the configuration space $\operatorname{comp}\left(\omega_{0}\right) / \mathcal{G}^{r+1}\left(\omega_{0}\right)$. A special feature of the theory on open manifolds is the fact that the isotropy group of a connection is discrete in $\mathcal{G}^{r+1}\left(\omega_{0}\right)$. We cannot present here all proofs in detail which are contained in [13]. The general procedure has been essentially modelled in [14].

Up to a great part we follow the schedule of [14], but with modified analytic arguments. To obtain closed image properties, we must add the spectral condition that the essential spectrum of a certain Laplace operator starts away from zero.

2. The space of connections. We assume $\left(M^{n}, g\right)$ open, complete with $(I)$ and $\left(B_{k}\right)$. As is well known, there is no obstruction against the existence of a metric $g$ with $(I)$ and $\left(B_{k}\right), 0 \leq k \leq \infty$. Let $(E, h)$ be a Riemannian vector bundle with metric connection $\nabla^{E}$. Additionally to $(I)$ and $\left(B_{k}\right)$ for $\left(M^{n}, g\right)$ we define the condition

$\left(B_{k}\left(E, \nabla^{E}\right)\right)$

$\left|\left(\nabla^{E}\right)^{i} R^{E}\right| \leq C_{i}, 0 \leq i \leq k$.

The Levi-Civita connection $\nabla^{g}$ and $\nabla^{E}$ induce metric connections $\nabla$ in all tensor bundles with values in $E, T_{r}^{q}(M) \otimes E$. Denote by $\Omega^{q}(E)$ the space of $E$-valued $q$-forms, $\Omega^{q}(E)=$ $C^{\infty}\left(\bigwedge^{q} T^{*} M \otimes E\right)$. Now we define several Sobolev spaces and restrict ourselves to the case of $E$-valued $q$-forms. The case of $E$-valued tensor is quite analogous and can be considered as $\Omega^{0}\left(T_{q}^{r} \otimes E\right)$.

Let for $1 \leq p<\infty, r \geq 0$

$$
\begin{aligned}
& \Omega_{r}^{q, p}(E):=\left\{\left.\sigma \in \Omega^{q}(E)|| \sigma\right|_{p, r}:=\left(\int_{M} \sum_{i=0}^{r}\left|\nabla^{i} \sigma\right|_{x}^{p} d \operatorname{vol}_{x}(g)\right)^{1 / p}<\infty\right\}, \\
& \bar{\Omega}^{q, p, r}(E)=\text { completion of } \Omega_{r}^{q, p}(E) \text { with respect to }||_{p, r}, \\
& \widehat{\Omega}^{q, p, r}(E)=\text { completion of } C_{0}^{\infty}\left(\bigwedge T^{*} M \otimes E\right) \text { with respect to }||_{p, r} \text { and } \\
& \Omega^{q, p, r}(E)=\left\{\sigma \mid \sigma \text { measurable distributional } q \text {-form such that }|\sigma|_{p, r}<\infty\right\} .
\end{aligned}
$$

Moreover define for $m \geq 0$

$$
\begin{aligned}
& { }_{m}^{b} \Omega^{q}(E)=\left\{\left.\sigma \in \Omega^{q}(E)\left|{ }^{b, m}\right| \sigma\left|:=\sum_{i=0}^{m}\right| \nabla^{i} \sigma\right|_{x}<\infty\right\}, \\
& { }^{b, m} \Omega^{q}(E)=\text { completion of }{ }_{m}^{b} \Omega^{q}(E) \text { with respect to }{ }^{b, m}|| \text { and } \\
& { }_{b, m} \widehat{\Omega}^{q}(E)=\text { completion of } C_{0}^{\infty}\left(\bigwedge^{q} T^{*} M \otimes E\right) \text { with respect to }{ }^{b, m}|| .
\end{aligned}
$$

Then

$$
{ }^{b, m} \Omega^{q}(E)=\left\{\sigma \mid \sigma \text { is a } C^{m} \text {-form with }{ }^{b, m}|\sigma|<\infty\right\} .
$$

There are natural inclusions

$$
\begin{gathered}
{ }^{b, m} \widehat{\Omega}^{q}(E) \subsetneq{ }_{m}^{b} \Omega^{q}(E) \\
\widehat{\Omega}^{q, p, r}(E) \subseteq \bar{\Omega}^{q, p, r}(E) \subseteq \Omega^{q, p, r}(E) .
\end{gathered}
$$

Proposition 2.1. Assume $\left(M^{n}, g\right)$ open, complete with $(I)$ and $\left(B_{k}\right)$. Then

$$
\widehat{\Omega}^{q, p, r}(E)=\bar{\Omega}^{q, p, r}(E)=\Omega^{q, p, r}(E) .
$$


Theorem 2.2. Assume $\left(M^{n}, g\right)$ open, complete with $(I)$ and $\left(B_{0}\right), r>n / p+m$. Then there exists a continuous embedding

$$
\begin{gathered}
\Omega^{q, p, r}(E) \hookrightarrow^{b, m} \Omega^{q}(E) . \\
{ }^{b, m}|\sigma| \leq D \cdot|\sigma|_{p, r} .
\end{gathered}
$$

Theorem 2.3. Assume $\left(M^{n}, g\right),\left(E_{i}, h_{i}, \nabla^{E_{i}}\right) \rightarrow M, i=1,2$, with $(I)$ and $\left(B_{k}\right)$, $k \geq r_{1}, r_{2} \geq r \geq 0$. If $r=0$ assume

$$
\left\{\begin{array}{l}
r-\frac{n}{p}<r_{1}-\frac{n}{p_{1}} \\
r-\frac{n}{p}<r_{2}-\frac{n}{p_{2}} \\
r-\frac{n}{p} \leq r_{1}-\frac{n}{p_{1}}+r_{2}-\frac{n}{p_{2}} \\
\frac{1}{p} \leq \frac{1}{p_{1}}+\frac{1}{p_{2}}
\end{array}\right\} \text { or }\left\{\begin{array}{r}
r-\frac{n}{p} \leq r_{1}-\frac{n}{p_{1}} \\
0<r_{2}-\frac{n}{p_{2}} \\
\frac{1}{p} \leq \frac{1}{p_{1}}
\end{array}\right\} \text { or }\left\{\begin{array}{c}
0<r_{1}-\frac{n}{p_{1}} \\
r-\frac{n}{p} \leq r_{2}-\frac{n}{p_{2}} \\
\frac{1}{p} \leq \frac{1}{p_{2}}
\end{array}\right\} \text {. }
$$

If $r>0$ assume $\frac{1}{p} \leq \frac{1}{p_{1}}+\frac{1}{p_{2}}$ and

$$
\left\{\begin{array}{l}
r-\frac{n}{p}<r_{1}-\frac{n}{p_{1}} \\
r-\frac{n}{p}<r_{2}-\frac{n}{p_{2}} \\
r-\frac{n}{p} \leq r_{1}-\frac{n}{p_{1}}+r_{2}-\frac{n}{p_{2}}
\end{array}\right\} \text { or }\left\{\begin{array}{l}
r-\frac{n}{p} \leq r_{1}-\frac{n}{p_{1}} \\
r-\frac{n}{p} \leq r_{2}-\frac{n}{p_{2}} \\
r-\frac{n}{p}<r_{1}-\frac{n}{p_{1}}+r_{2}-\frac{n}{p_{2}}
\end{array}\right\} \text {. }
$$

Then there exists a continuous embedding

$$
\Omega^{p_{1}, r_{1}}\left(E_{1}\right) \otimes \Omega^{p_{2}, r_{2}}\left(E_{2}\right) \hookrightarrow \Omega^{p, r}\left(E_{1} \otimes E_{2}\right),
$$

where we write $\Omega^{p, r} \equiv \Omega^{0, p, r}$.

The proofs of Proposition 2.1 and Theorem 2.2 are contained in [3], of Theorem 2.3 in $[10]$.

Now we are able to define for $\mathcal{C}_{P}\left(B_{k}\right)$ a uniform structure which allows us to complete this space. Let $\delta>0,1<p<\infty, k \geq r>n / p+1$, for $\eta \in \Omega^{1}\left(\mathbf{g}_{P}\right)$

$$
|\eta|_{\omega, p, r}:=\left(\int_{M} \sum_{i=0}^{r}\left|\left(\nabla^{\omega}\right)^{i} \eta\right|_{x}^{p} d \operatorname{vol}_{x}(g)\right)^{1 / p}
$$

and set

$$
V_{\delta}:=\left\{\left(\omega, \omega_{1}\right) \in \mathcal{C}_{P}\left(B_{k}\right)^{2}|| \omega-\left.\omega_{1}\right|_{\omega, p, r}<\delta\right\} .
$$

THEOREM 2.4. $\mathcal{B}=\left\{V_{\delta}\right\}_{\delta>0}$ is a basis for a metrizable uniform structure $\mathcal{U}^{p, r}\left(\mathcal{C}_{P}\left(B_{k}\right)\right)$ on $\mathcal{C}_{P}\left(B_{k}\right)$.

Denote by $\mathcal{C}_{P}^{p, r}\left(B_{k}\right)$ the completion with respect to this uniform structure and by $\operatorname{comp}(\omega)$ the component of $\omega$ in $\mathcal{C}_{P}^{p, r}\left(B_{k}\right)$. 


\section{THEOREM 2.5.}

a. $\operatorname{comp}(\omega)=\omega+\Omega^{1, p, r}\left(\mathbf{g}_{P}, \nabla^{\omega}\right)$.

b. $\mathcal{C}_{P}\left(B_{k}\right)$ has a representation as a topological sum,

$$
\mathcal{C}_{P}\left(B_{k}\right)=\sum_{i \in I} \operatorname{comp}\left(\omega_{i}\right)=\sum_{i \in I}\left(\omega_{i}+\Omega^{1, p, r}\left(\mathbf{g}_{P}, \nabla^{\omega_{i}}\right)\right) .
$$

(In the sequel we restrict ourselves to the case $p=2$.)

Denote by $\mathcal{C}_{P}\left(B_{k}\right.$, fin) the set of all connections $\mathcal{C}_{P}\left(B_{k}\right)$ such that

$$
\mathcal{Y} \mathcal{M}(\omega)=\frac{1}{2} \int_{M}\left|R^{\omega}\right|^{2} d \operatorname{vol}_{x}(g)<\infty .
$$

Then $i: \mathcal{C}_{P}\left(B_{k}\right.$, fin $) \hookrightarrow \mathcal{C}_{P}\left(B_{k}\right)$ induces a uniform structure $i^{-1}\left(\mathcal{U}^{p, r}\left(\mathcal{C}_{P}\left(B_{k}\right)\right)\right)$ and defines a completion $\mathcal{C}_{P}^{2, r}\left(B_{k}\right.$, fin).

TheOREM 2.6. $\mathcal{C}_{P}^{2, r}\left(B_{k}\right.$, fin) has a representation as a topological sum,

$$
\mathcal{C}_{P}^{2, r}\left(B_{k}, \text { fin }\right)=\sum_{j \in J} \operatorname{comp}\left(\omega_{j}\right)=\sum_{j \in J}\left(\omega_{j}+\Omega^{1,2, r}\left(\mathbf{g}_{P}, \nabla^{\omega_{j}}\right)\right) .
$$

The proofs of Theorems 2.4-2.6 are contained in [4] and use essentially Proposition 2.1 and Theorems 2.2 and 2.3 .

Remark. The proof that $\operatorname{comp}(\omega)=\omega+\Omega^{1,2, r}\left(\mathbf{g}_{P}, \nabla^{\omega}\right)$ essentially uses the fact that $\omega^{\prime} \in \operatorname{comp}(\omega)$ implies

$$
\Omega^{1,2, r}\left(\mathbf{g}_{P}, \nabla^{\omega}\right)=\Omega^{1,2, r}\left(\mathbf{g}_{P}, \nabla^{\omega^{\prime}}\right)
$$

as equivalent Sobolev spaces. This proof is nontrivial. Another difficulty arises from the fact that the elements of $\mathcal{C}_{P}^{2, r}\left(B_{k}\right.$, fin) are only $C^{1}$ but smooth elements are dense. At the first instance, for $\omega$ non-smooth $\left(\nabla^{\omega}\right)^{i}$ does not make sense for $i>2$. But if $\omega^{\prime}$ is a smooth connection in an $\varepsilon$-neighbourhood of $\omega$ then we define

$$
\nabla^{\omega}:=\nabla^{\omega^{\prime}}+\nabla^{\omega}-\nabla^{\omega^{\prime}} .
$$

It is easy to see that $\left(\nabla^{\omega^{\prime}}+\left(\nabla^{\omega}-\nabla^{\omega^{\prime}}\right)\right)^{i}$ makes sense for any $i$.

In the sequel, we consider one fixed component $\operatorname{comp}\left(\omega_{0}\right) \subset \mathcal{C}_{P}^{2, r}\left(B_{k}\right.$, fin) and write $\mathcal{C}^{r}\left(\omega_{0}\right) \equiv \operatorname{comp}\left(\omega_{0}\right)$.

3. The gauge group. There are three equivalent definitions of the gauge group,

$$
\begin{aligned}
& \mathcal{G}=\left\{\varphi: P \stackrel{\simeq}{\longrightarrow} P \mid \varphi \text { covers id }_{M} \text { and } \varphi(p \cdot g)=\varphi(p) \cdot g\right\}, \\
& \widehat{\mathcal{G}}=\left\{\widehat{\varphi}: P \longrightarrow G \mid \widehat{\varphi}(p \cdot g)=g^{-1} \widehat{\varphi}(p) g\right\}, \\
& \widetilde{\mathcal{G}}=C^{\infty}\left(P \times_{G} G\right),
\end{aligned}
$$

where $G$ acts on itself by means of the inner automorphism Ad.

$\mathcal{G}, \widehat{\mathcal{G}}$ and $\widetilde{\mathcal{G}}$ are isomorphic: $\varphi \in \mathcal{G}$ given defines $\widehat{\varphi}$ by $\varphi(p)=p \cdot \widehat{\varphi}(p)$ and $\widetilde{\varphi}(x)=$ $\left[(p, \widehat{\varphi}(p)] \in \widetilde{\mathcal{G}}\right.$, where $p \in \pi^{-1}(x)$. Any Sobolev construction for one of the three groups induces by extending the isomorphism above such a construction for the other three groups. We concentrate here to the group $\mathcal{G}^{r+1}\left(\omega_{0}\right)=\widehat{\mathcal{G}}^{r+1}\left(\omega_{0}\right)$ adapted to $\mathcal{C}^{r}\left(\omega_{0}\right)$ and proceed 
as follows. First we construct $\mathcal{G}^{r+1}\left(\omega_{0}\right)$ as topological group. Then we endow a neighbourhood of the unit element with the structure of a local Lie group, using CampbellHausdorff series. According to a standard theorem, $\mathcal{G}^{r+1}\left(\omega_{0}\right)$ then is a Hilbert-Lie group. To do this, we have to estimate the norm of the commutator by the product of the norm of the factors. This is the key step of our construction. Unfortunately, in general $\mathcal{G}^{r+1}\left(\omega_{0}\right)$ does not satisfy the second axiom of countability. Therefore we have to consider later on a smaller group $\mathcal{G}_{\bullet}^{r+1}\left(\omega_{0}\right)$. We have to define the elements of the completed gauge group $\mathcal{G}^{r+1}\left(\omega_{0}\right)$ and to introduce a natural, suitable Sobolev topology. First we motivate our definition and start with the non-completed group. $\mathcal{G}$ acts on $\mathcal{C}_{P}$ from the left as follows:

$$
\varphi \omega:=\omega \circ d\left(\varphi^{-1}\right) \text {. }
$$

In terms of $\widehat{\varphi} \in \widehat{\mathcal{G}}$ this means

$$
\varphi \omega=\operatorname{Ad}(\widehat{\varphi}) \omega+\left(\widehat{\varphi}^{-1}\right)^{*} \theta
$$

where $\theta$ denotes the Maurer-Cartan form of $G$. This implies $\varphi \omega-\omega=(\varphi \omega-\omega) \circ\left(\operatorname{proj}_{v}^{\omega}+\right.$ $\left.\operatorname{proj}_{h}^{\omega}\right)=(\varphi \omega-\omega) \circ \operatorname{proj}_{h}^{\omega}$ since $\varphi \omega-\omega$ is horizontal. Hence

$$
\varphi \omega-\omega=(\varphi \omega-\omega) \circ \operatorname{proj}_{h}^{\omega}=\left(\widehat{\varphi}^{-1}\right)^{*} \theta \circ \operatorname{proj}_{h}^{\omega} .
$$

Rem ark. It is easy to see that $\left(\widehat{\varphi}^{-1}\right)^{*} \theta \circ \operatorname{proj}_{h}^{\omega}=-d \mathrm{R}_{\widehat{\varphi}}^{-1} \circ \widehat{\varphi}$.

We define

$$
\nabla^{\omega} \widehat{\varphi}:=\left(\widehat{\varphi}^{-1}\right)^{*} \theta \circ \operatorname{proj}_{h}^{\omega}=-d \mathrm{R}_{\widehat{\varphi}}^{-1} \circ \widehat{\varphi}
$$

Then

$$
\varphi \omega=\omega+\nabla^{\omega} \widehat{\varphi} .
$$

Similarly as the groups $\mathcal{G}, \widehat{\mathcal{G}}, \widetilde{\mathcal{G}}$, we denote at the Lie algebra level by $\widehat{X}: P \rightarrow \mathbf{g}$ vector fields with

$$
\widehat{X}_{p g}=\operatorname{Ad}\left(g^{-1}\right) X_{p}
$$

and by $\widetilde{X}$ sections of $\mathbf{g}_{P} . \widehat{X}$ and $\widetilde{X}$ correspond to each other.

Let $\omega_{0} \in \mathcal{C}\left(B_{k}\right.$, fin $)$ smooth. Then $\left(P, g_{\omega_{0}}\right)$ is a manifold satisfying $(I)$ and $\left(B_{k}\right)$, where $g_{\omega_{0}}$ is the Kaluza-Klein metric,

$$
g_{\omega_{0}}(X, Y):=g_{M}\left(\pi_{*} X, \pi_{*} Y\right)+\left\langle\omega_{0}(X), \omega_{0}(Y)\right\rangle_{\text {Killing }} .
$$

This has been proven in [4]. We say $\widehat{\varphi}: P \rightarrow G$ is bounded up to order $r$ if

$$
{ }^{b, r-1}|d \widehat{\varphi}|:=\sum_{i=0}^{r-1} \sup _{p \in P}\left|\nabla^{i} d \widehat{\varphi}\right|_{x}<\infty .
$$

Here $d \widehat{\varphi}$ is considered as a section of $T^{*} P \otimes \widehat{\varphi}^{*} T G$, endowed with the induced connection. Then the manifold $\Omega^{r}(P, G)$ of maps is well defined (cf. [5], [11]). $f \in \Omega^{r}(P, G)$ if and only if for any $\varepsilon>0$ there exists $f_{0} \in \Omega^{\infty}(P, G)$ with ${ }^{b, r-1}\left|d f_{0}\right|<\infty$ and $X \in \Omega^{r}\left(f_{0}^{*} T G, f_{0}^{*} \nabla^{G}\right)$ with $|X|_{r}<\varepsilon$ such that

$$
f=\exp X=\exp _{f_{0}} X \circ f_{0} .
$$

If $k \geq r>n / 2+1$ then $f \in C^{1}$, according to the Sobolev embedding theorem.

In the sequel, we assume $\left(M^{n}, g\right)$ open complete with $(I)$ and $\left(B_{k}\right), k \geq r>n / 2+1$ and consider $\mathcal{C}^{r}\left(\omega_{0}\right) \equiv \operatorname{comp}\left(\omega_{0}\right) \subset \mathcal{C}^{2, r}\left(B_{k}\right.$, fin $)$. 
Proposition 3.1. Assume $\nabla^{\omega_{0}} \widehat{\varphi} \in \Omega^{1,2, r}\left(\mathbf{g}_{P}, \nabla^{\omega_{0}}\right), \omega \in \mathcal{C}^{r}\left(\omega_{0}\right)$. Then $\varphi \omega \in \mathcal{C}^{r}\left(\omega_{0}\right)$.

Proof. $\omega=\omega_{0}+\eta, \eta \in \Omega^{1,2, r}\left(\mathbf{g}_{P}, \nabla^{\omega_{0}}\right)$

$$
\varphi \omega=\varphi\left(\omega_{0}+\eta\right)=\omega_{0}+\eta+\nabla^{\omega_{0}+\eta} \widehat{\varphi} .
$$

On the other hand,

$$
\varphi \omega=\varphi \omega_{0}+\operatorname{Ad}(\widehat{\varphi}) \eta=\omega_{0}+\nabla^{\omega_{0}} \widehat{\varphi}+\operatorname{Ad}(\widehat{\varphi})
$$

hence

$$
\nabla^{\omega_{0}+\eta} \widehat{\varphi}=\nabla^{\omega_{0}} \widehat{\varphi}+(\operatorname{Ad}(\widehat{\varphi})-I) \eta .
$$

We are done if we could show

$$
|\operatorname{Ad}(\widehat{\varphi}) \eta|_{\omega_{0}, r}<\infty .
$$

Since $\nabla^{\omega_{0}} \widehat{\varphi} \in \Omega^{1,2, r}\left(\mathbf{g}_{P}, \nabla^{\omega_{0}}\right)$ and $\varphi \omega_{0} \in \mathcal{C}^{r}\left(\omega_{0}\right)$ are equivalent, $\omega_{0}$ and $\varphi \omega_{0}$ generate equivalent Sobolev norms. Hence it suffices to show

$$
|\operatorname{Ad}(\widehat{\varphi}) \eta|_{\varphi \omega_{0}, r}<\infty
$$

But an easy straightforward calculation shows

$$
|\operatorname{Ad}(\widehat{\varphi}) \eta|_{\varphi \omega_{0}, r}=|\eta|_{\omega_{0}, r}<\infty .
$$

This motivates the following definition. Let $\mathcal{G}^{r+1}\left(\omega_{0}\right)$ be the set of all $\widehat{\varphi} \in \Omega^{r+1}(P, G)$ such that

(i) $\widehat{\varphi}(p g)=g^{-1} \widehat{\varphi}(p) g$

(ii) $\nabla^{\omega_{0}} \widehat{\varphi} \in \Omega^{1,2, r}\left(\mathbf{g}_{P}, \nabla^{\omega_{0}}\right)$.

Proposition 3.2. $\mathcal{G}^{r+1}\left(\omega_{0}\right)$ forms a group under pointwise multiplication.

Proof. First we have to show that $\Omega^{r+1}(P, G)$ forms a group under pointwise multiplication, i.e. given any $\varepsilon>0$ and $f, g \in \Omega^{r+1}(P, G)$ there exists $h_{0} \in C^{\infty}(P, G)$, ${ }^{b, r}\left|d h_{0}\right|<\infty, Z \in \Omega^{r+1}\left(h_{0}^{*} T G\right),|Z|_{r+1}<\varepsilon$ such that $f \cdot g=\exp Z \equiv_{h_{0}} Z \circ h_{0}$. This is highly nontrivial and follows from [5], [11]. Next we show that $\mathcal{G}^{r+1}\left(\omega_{0}\right)$ is a subgroup. Condition (i) is trivial. For (ii) we have to show:

a. $\widehat{\varphi}, \widehat{\psi} \in \mathcal{G}^{r+1}\left(\omega_{0}\right)$ implies $\left|\nabla^{\omega_{0}}(\widehat{\psi} \widehat{\varphi})\right|_{\omega_{0}, r}<\infty$.

b. $\widehat{\varphi} \in \mathcal{G}^{r+1}\left(\omega_{0}\right)$ implies $\left|\nabla^{\omega_{0}}\left(\widehat{\varphi}^{-1}\right)\right|_{\omega_{0}, r}<\infty$.

The first assertion follows from

$$
\nabla^{\omega_{0}}(\widehat{\psi} \widehat{\varphi})=\operatorname{Ad}(\widehat{\psi}) \nabla^{\omega_{0}} \widehat{\varphi}+\nabla^{\omega_{0}} \widehat{\psi}
$$

and the second from

$$
\nabla^{\omega_{0}}\left(\widehat{\varphi}^{-1}\right)=-\operatorname{Ad}\left(\widehat{\varphi}^{-1}\right) \nabla^{\omega_{0}} \widehat{\varphi} .
$$

Here (3.2) follows from (3.1) by setting $\widehat{\psi}=\widehat{\varphi}^{-1}$.

The next step is the introduction of a suitable Sobolev topology. We have to establish a filter basis $\mathcal{B}=\mathcal{B}(e)$ for $e \in \mathcal{G}^{r+1}\left(\omega_{0}\right)$ satisfying the following conditions:

(i) If $U \in \mathcal{B}$ then there exists $V \in \mathcal{B}$ such that $V V^{-1} \subseteq U$.

(ii) If $U \in \mathcal{B}$ then there exists $V \in \mathcal{B}$ such that $V^{-1} \subseteq U$.

(iii) $e \in U$ for each $U \in \mathcal{B}$. 
(iv) If $g \in \mathcal{G}^{r+1}\left(\omega_{0}\right)$ and $U \in \mathcal{B}$ then there exists $V \in \mathcal{B}$ such that $V \subseteq g U g^{-1}$.

The proof of the existence of $\mathcal{B}$ with (i)-(iv) shall be prepared by a series of propositions.

Observe that $\Omega^{q}\left(\mathbf{g}_{P}\right)$ and the space $\Omega^{q}(P, \mathbf{g})^{G} \subset \Omega^{q}(P, \mathbf{g})$ of $\mathbf{g}$-valued $G$-invariant $q$-forms on $P$ are isomorphic. Let $s, t \in \Omega^{0}(P, \mathbf{g})$. Pointwise commutators define $[s, t] \in$ $\Omega^{0}(P, \mathbf{g})$. Define for $\sigma \in \Omega^{1}(P, \mathbf{g})$ and $X \in \Omega^{0}(T P) \equiv \Omega(T P)$

$$
\begin{aligned}
& {[\sigma \wedge s](X):=[\sigma(X), s]} \\
& {[s \wedge \sigma](X):=[s, \sigma(X)] .}
\end{aligned}
$$

$\omega \in \mathcal{C}$ defines a covariant differential $D^{\omega}$ :

$$
\Omega^{0}\left(\mathbf{g}_{P}\right) \simeq \Omega(P, \mathbf{g})^{G} \stackrel{D^{\omega}}{\longrightarrow} \Omega^{1}(P, \mathbf{g})_{h}^{G} \simeq \Omega^{1}\left(\mathbf{g}_{P}\right),
$$

where ()$_{h}$ denotes horizontal forms.

Lemma 3.3. For $s \in \Omega(P, \mathbf{g})^{G} \simeq \Omega^{0}\left(\mathbf{g}_{P}\right)$,

$$
D^{\omega} s=d s+[\omega \wedge s] .
$$

Here one writes with a basis $\left\{A_{i}\right\}$ of $\mathbf{g}, s=s^{i} A_{i}, d s=d s^{i} \otimes A_{i}$. For a proof see [12].

$D^{\omega}$ satisfies the following product rule.

Lemma 3.4. For $s, t \in \Omega(P, \mathbf{g})^{G}$,

$$
D^{\omega}[s, t]=\left[D^{\omega} s \wedge t\right]+\left[s \wedge D^{\omega} t\right] .
$$

The proof is an easy calculation with the use of (3.3).

R e mark. For higher derivatives, a similar simple formula is not available. It holds only up to permutation in tensor products (cf. [9]). But for norm estimates a weaker product rule is available. Consider the sequence

$$
\Omega^{0}\left(\mathbf{g}_{P}\right) \stackrel{D^{\omega}}{\longrightarrow} \Omega^{1}\left(\mathbf{g}_{P}\right) \stackrel{\nabla^{\omega}}{\longrightarrow} \Omega^{0}\left(\left(T^{*} M\right)^{\otimes 2} \otimes \mathbf{g}_{P}\right) \stackrel{\nabla^{\omega}}{\longrightarrow} \cdots .
$$

Proposition 3.5. For $s, t \in \Omega^{0}\left(\mathbf{g}_{P}\right), X_{1}, \ldots, X_{u} \in \Omega(T M) \equiv \Omega^{0}(T M)$,

$$
\nabla_{X_{u} \cdots X_{1}}^{u}[s, t]=\sum_{i=0}^{u} \sum_{u \geq \alpha_{i} \geq \cdots \geq \alpha_{1} \geq 1}\left[\nabla_{X_{\alpha_{i}} \cdots X_{\alpha_{1}}}^{i} s, \nabla_{X_{u} \cdots \widehat{X}_{\alpha_{i}} \cdots \widehat{X}_{\alpha_{1}} \cdots X_{1}}^{u-i} t\right] .
$$

This can be proven by an easy induction, starting with Lemma 3.4.

THEOREM 3.6. Let $\left(M^{n}, g\right)$ be open, complete with $(I)$ and $\left(B_{k}\right), \omega_{0}$ with $\left(B_{k}\right), k \geq$ $r>n / 2+1$. Then there exists a $\Xi \in \mathbb{R}, \Xi>0$, such that for all $\widetilde{X}, \widetilde{Y} \in \Omega^{0,2, r}\left(\mathbf{g}_{P}, \nabla^{\omega_{0}}\right) \equiv$ $\mathbf{g}_{P}^{r}\left(\omega_{0}\right)$,

$$
|[\tilde{X}, \tilde{Y}]|_{\omega_{0}, r} \leq \Xi \cdot|\widetilde{X}|_{\omega_{0}, r} \cdot|\widetilde{Y}|_{\omega_{0}, r} .
$$

Proof. For $A, B \in \mathbf{g}$,

$$
|[A, B]| \leq c \cdot|A| \cdot|B| .
$$

Proposition 3.5 then implies for $s, t \in \Omega^{0}\left(\mathbf{g}_{P}\right)$

$$
\left|\nabla^{u}[s, t]\right|_{x}^{2} \leq C \cdot \sum_{i=0}^{u}\left(\left|\nabla^{i} s\right|_{x} \cdot\left|\nabla^{u-i} t\right|_{x}\right)^{2} .
$$


The module structure theorem 2.3 finally yields the assertion.

Re mark. (3.4) is the key inequality for establishing a Campbell-Hausdorff series in our case and for endowing $\mathcal{G}^{r+1}\left(\omega_{0}\right)$ with the structure of a local Lie group. For this, we recall some definitions.

Let $G$ a Lie group with Lie algebra $\mathbf{g}$ and exponential exp. Assume $\mathbf{g}$ to be endowed with a norm || such that there exists a $\Xi \geq 1$ with

$$
|[x, y]| \leq \Xi \cdot|x| \cdot|y|
$$

Set

$$
\mathrm{CH}(x, y):=\sum_{k=1}^{\infty} \sum_{\mu, \nu \in \mathbb{N}^{k}} \frac{(-1)^{k-1}}{k} \frac{1}{\mu_{1}+\nu_{1}+\cdots+\mu_{k} \nu_{k}} \frac{\left[x^{\mu_{1}} y^{\nu_{1}} \cdots x^{\mu_{k}} y^{\nu_{k}}\right]}{\mu_{1} ! \nu_{1} ! \cdots \mu_{k} ! \nu_{k} !}
$$

where

$$
\left[x^{\mu_{1}} y^{\nu_{1}} \cdots x^{\mu_{k}} y^{\nu_{k}}\right]:=\underbrace{[[x, x], x], \cdots x]}_{\mu_{1} \text { times }}, \underbrace{y], \cdots, y]}_{\nu_{1} \text { times }}, \cdots, \underbrace{x], \cdots, x]}_{\mu_{k} \text { times }}, \underbrace{y], \cdots, y]}_{\nu_{k} \text { times }} .
$$

Theorem 3.7. Assume (3.3). Then there exists a constant $C(\Xi)$ such that with $U:=$ $\{x \in \mathbf{g}|| x \mid<C(\Xi)\}, \mathrm{CH}(\cdot, \cdot)$ becomes an analytic mapping $U \times U \rightarrow \mathbf{g}$ and

$$
\exp (x) \exp (y)=\exp (\mathrm{CH}(x, y))
$$

This is a classical result.

We do not recall the definition of analytic mapping between Banach spaces which is contained in [1].

TheOREM 3.8. Let $L$ be a complete normed Lie algebra satisfying (3.5). Assume $\alpha \in \mathbb{R}$, $0<\alpha \leq 1 /(3 \Xi) \cdot \log 3 / 2$. Set $L_{\alpha}:=\{x \in L|| x \mid<\alpha\}, \Theta:=\left\{(x, y) \in L_{\alpha} \times L_{\alpha} \mid \mathrm{CH}(x, y) \in\right.$ $\left.L_{\alpha}\right\}$ and define $m=\mathrm{CH} \mid \Theta$. Then

(i) $\Theta$ is open in $L_{\alpha} \times L_{\alpha}$ and $m$ analytic;

(ii) $x \in L_{\alpha}$ implies $(0, x),(x, 0) \in \Theta$ and $m(0, x)=m(x, 0)=0$;

(iii) $x \in L_{\alpha}$ implies $-x \in L_{\alpha},(x,-x),(-x, x) \in \Theta$ and $m(x,-x)=m(-x, x)=0$;

(iv) $x, y, z \in L_{\alpha},(x, y),(m(x, y), z),(y, z),(x, m(y, z)) \in \Theta$ implies $m(m(x, y), z)=$ $m(x, m(y, z))$;

(v) $x, y \in L_{\alpha}$ implies $|\mathrm{CH}(x, y)| \leq-1 / \Xi \cdot \log (2-\exp \Xi(|x|+|y|))$.

For a proof see $[2]$.

Definition. A couple $(\Gamma, e, \Theta, m)$ is called a local Lie group over $\mathbb{R}$ if it satisfies the following conditions:

(i) $\Gamma$ is an analytic manifold modelled by a real Hilbert space;

(ii) $e \in \Gamma$;

(iii) $\theta: \Gamma \rightarrow \Gamma$ is an analytic mapping;

(iv) $m$ is an analytic mapping from an open subset $\Omega \subset \Gamma \times \Gamma$ into $\Gamma$;

(v) $m(e, g), m(g, e) \in \Gamma$ and $m(e, g)=m(g, e)=g$ for all $g \in \Gamma$;

(vi) $(g, \theta(g)),(\theta(g), g) \in \Omega$ and $m(g, \theta(g))=m(\theta(g), g)=e$ for all $g \in \Gamma$; 
(vii) assume $g, h, k \in \Gamma$ arbitrary, $(g, h),(h, k),(m(g, h), k),(g, m(h, k)) \in \Omega$, then $m(m(g, h), k)=m(g, m(h, k))$.

Corollary 3.9. Assume $L_{\alpha}, m$ as in Theorem 3.8. Then with $i: L_{\alpha} \rightarrow L_{\alpha}, x \mapsto-x$, $\left(L_{\alpha}, 0, i, m\right)$ is a local Lie group.

Now we are able to endow $\mathcal{G}^{r+1}\left(\omega_{0}\right)$ with a topology such that there arises a topological and even a Hilbert-Lie group.

We define a neighbourhood filter basis of $e \in \mathcal{G}^{r+1}\left(\omega_{0}\right)$ as follows. Assume $0<\varepsilon_{0}<$ $\min \left\{D \cdot r_{\text {inj }}(M), 1 /(3 \Xi) \cdot \log 3 / 2\right\}$. ( $D$ comes from the Sobolev embedding theorem 2.2.) Set for $\varepsilon$

$$
U_{\varepsilon}:=\left\{\left.\exp \widehat{X}|| \widetilde{X}\right|_{\omega_{0}, r+1}<\varepsilon\right\} .
$$

THEOREM 3.10. $\mathcal{B}=\left\{U_{\varepsilon}\right\}_{0<\varepsilon<\varepsilon_{0}}$ is a filter basis for the neighbourhood filter of e such that $\mathcal{G}^{r+1}\left(\omega_{0}\right)$ becomes a topological group.

Proof. First we have to show $\exp \widehat{X} \in \mathcal{G}^{r+1}\left(\omega_{0}\right), \tilde{X} \in \mathbf{g}_{P}^{r+1}\left(\omega_{0}\right)$. This follows immediately from

$$
\nabla^{\omega_{0}} \exp \widehat{X}=\sum_{m=0}^{\infty}(-1)^{m+1} \frac{\operatorname{ad}(\tilde{X})^{m}}{(m+1) !}\left(\nabla^{\omega_{0}} \tilde{X}\right)
$$

Moreover, the map $\widetilde{X} \mapsto \nabla^{\omega_{0}} \exp \widehat{X}$ is even analytic. We have to establish properties (i)-(iv) for $\mathcal{B}$ (which were formulated after the proof of Proposition 3.2).

(i) Choose $\varepsilon^{\prime}<\min \left\{\varepsilon_{0}, 1 /(2 \Xi) \cdot \log 2-\exp (-1 /(2 \Xi) \cdot \varepsilon)\right\}$. Using (v) of Theorem 3.8, we see immediately $U_{\varepsilon^{\prime}} U_{\varepsilon^{\prime}} \subseteq U_{\varepsilon}$.

(ii) For $0<\varepsilon<\varepsilon_{0}, U_{\varepsilon}^{-1}=U_{\varepsilon}$.

(iii) $e=\exp 0$.

(iv) Here we have to show: Given any $\varphi \in \mathcal{G}^{r+1}\left(\omega_{0}\right), 0<\varepsilon<\varepsilon_{0}$, there exists $0<\varepsilon^{\prime}<\varepsilon_{0}$ such that $U_{\varepsilon^{\prime}} \subseteq \widehat{\varphi} U_{\varepsilon} \widehat{\varphi}^{-1}$. For this we consider the map $\mathcal{G}^{r+1}\left(\omega_{0}\right) \rightarrow$ $\mathcal{L}\left(\mathbf{g}_{P}^{r+1}\left(\omega_{0}\right), \mathbf{g}_{P}^{r+1}\left(\omega_{0}\right)\right), \widehat{\varphi} \mapsto \operatorname{Ad}\left(\widehat{\varphi}^{-1}\right)$. This map is well defined. Write $\widehat{\varphi}=\exp _{\hat{\varphi}_{0}} \widehat{Z} \cdot \widehat{\varphi}_{0}$. Then $\operatorname{Ad}\left(\widehat{\varphi}_{0}^{-1}\right)$ acts as bounded operator on $\mathbf{g}_{P}^{r+1}\left(\omega_{0}\right)$ according to the boundedness condition of $\widehat{\varphi}_{0}$. The same holds for $\operatorname{Ad}\left(\left(\exp _{\hat{\varphi}_{0}} \widehat{Z}\right)^{-1}\right)$ according to the module structure theorem. This is a highly nontrivial fact and we refer to [5]. Denote by $\left\|\operatorname{Ad}\left(\widehat{\varphi}^{-1}\right)\right\|_{\omega_{0}, r+1}$ the corresponding operator norm. Choose

$$
\varepsilon^{\prime}<\min \left\{\frac{\varepsilon}{\left\|\operatorname{Ad}\left(\widehat{\varphi}^{-1}\right)\right\|_{\omega_{0}, r+1}}, \varepsilon_{0}\right\} .
$$

Let $\exp \widehat{X} \in U_{\varepsilon^{\prime}}$ and choose $\tilde{Y}:=\operatorname{Ad}\left(\widehat{\varphi}^{-1}\right) \widetilde{X}$. Then

$$
\exp \widehat{X}=\exp \operatorname{Ad}(\widehat{\varphi}) \operatorname{Ad}\left(\widehat{\varphi}^{-1}\right) \widehat{X}=\exp \operatorname{Ad}(\widehat{\varphi}) \widehat{Y}=\widehat{\varphi} \exp \widehat{Y} \widehat{\varphi}^{-1} \in \widehat{\varphi} U_{\varepsilon} \widehat{\varphi}^{-1}
$$

if we can show $\exp \widehat{Y} \in U_{\varepsilon}$, i.e. $|\widetilde{Y}|_{\omega_{0}, r+1}<\varepsilon$. But

$$
\begin{aligned}
|\tilde{Y}|_{\omega_{0}, r+1} & =\left|\operatorname{Ad}\left(\widehat{\varphi}^{-1}\right) \tilde{X}\right|_{\omega_{0}, r+1} \leq\left\|\operatorname{Ad}\left(\widehat{\varphi}^{-1}\right)\right\|_{\omega_{0}, r+1}|\tilde{X}|_{\omega_{0}, r+1} \\
& \leq\left\|\operatorname{Ad}\left(\widehat{\varphi}^{-1}\right)\right\|_{\omega_{0}, r+1} \cdot \frac{\varepsilon}{\left\|\operatorname{Ad}\left(\widehat{\varphi}^{-1}\right)\right\|_{\omega_{0}, r+1}}=\varepsilon .
\end{aligned}
$$

We use the following well known 
THEOREM 3.11. Let $G$ be a topological group and $V \subset G$ an open neighbourhood of the unit element. Assume that $V$ is an analytic manifold and has the structure of a real local Lie group. Then $G$ is a Hilbert-Lie group.

Now we apply this to our situation. Let $U_{\varepsilon}$ be as in (3.7). $U_{\varepsilon}$ is homeomorphic to an open neighbourhood from the Hilbert space $\mathbf{g}_{P}^{r+1}\left(\omega_{0}\right)$. Then $L_{\varepsilon}:=\exp ^{-1}\left(U_{\varepsilon}\right)$ is a real local Lie group: Set for $\exp \widehat{X}, \exp \widehat{Y} \in U_{\varepsilon}$

$$
\begin{aligned}
m(\exp \widehat{X}, \exp \widehat{Y}) & :=\exp \mathrm{CH}(\exp \widehat{X}, \exp \widehat{Y})=\exp \widehat{X} \exp \widehat{Y} \\
\theta(\exp \widehat{X}) & :=\exp (-\widehat{X})=(\exp \widehat{X})^{-1} \\
e & :=0 .
\end{aligned}
$$

Hence we obtain

THEOREM 3.12. $\mathcal{G}^{r+1}\left(\omega_{0}\right)$ is a Hilbert-Lie group.

Remark. $\mathcal{G}^{r+1}\left(\omega_{0}\right)$ consists of $C^{1}$ elements $\widehat{\varphi}$. Sometimes we write $\varphi \in \mathcal{G}^{r+1}\left(\omega_{0}\right)$ which means the corresponding element $\varphi: P \stackrel{\simeq}{\longrightarrow} P$.

4. The action of the gauge group. Recall the action of $\mathcal{G}^{r+1}\left(\omega_{0}\right)$ on $\mathcal{C}^{r}\left(\omega_{0}\right)$ :

$$
\varphi \omega=\operatorname{Ad}(\widehat{\varphi}) \omega+\left(\widehat{\varphi}^{-1}\right)^{*} \theta=\omega+\nabla^{\omega} \widehat{\varphi} .
$$

Theorem 4.1. Assume $\left(M^{n}, g\right)$ open with $(I),\left(B_{k}\right), k \geq r+1>n / 2+2$. Then $\mathcal{G}^{r+1}\left(\omega_{0}\right)$ acts smoothly on $\mathcal{C}^{r}\left(\omega_{0}\right)$.

Proof. Let $\varphi \in \mathcal{G}^{r+1}\left(\omega_{0}\right), \omega=\omega_{0}+\eta \in \mathcal{C}^{r}\left(\omega_{0}\right), \eta \in \Omega^{1,2, r}\left(\mathbf{g}_{P}, \nabla^{\omega_{0}}\right)$ and $\widehat{X} \in$ $\mathbf{g}_{P}^{r+1}\left(\omega_{0}\right)$. Then

$$
(\widehat{\varphi} \exp \widehat{X})\left(\omega_{0}+\eta\right)=\omega_{0}+\nabla^{\omega_{0}}(\widehat{\varphi} \exp \widehat{X})+\operatorname{Ad}(\widehat{\varphi}) \operatorname{Ad}(\exp \widehat{X}) \eta .
$$

The assertion follows from the smoothness of the map

$$
(\widehat{X}, \eta) \mapsto \nabla^{\omega_{0}}(\widehat{\varphi} \exp \widehat{X})+\operatorname{Ad}(\widehat{\varphi}) \operatorname{Ad}(\exp \widehat{X}) \eta
$$

and the latter follows from the smoothness of the maps

$$
\begin{aligned}
(\widehat{X}, \eta) & \mapsto \nabla^{\omega_{0}}(\widehat{\varphi} \exp \widehat{X}) \\
(\widehat{X}, \eta) & \mapsto \operatorname{Ad}(\widehat{\varphi}) \operatorname{Ad}(\exp \widehat{X}) \eta .
\end{aligned}
$$

If the underlying manifold $M^{n}$ is compact then the action is proper. This is probably wrong in the open case. Nevertheless, one very important property of the action is preserved.

TheOREM 4.2. Assume $\left(M^{n}, g\right)$ open with $(I)$ and $\left(B_{k}\right), k \geq r>n / 2+2$. Then $\mathcal{G}^{r+1}\left(\omega_{0}\right)$ acts closed on $\mathcal{C}^{r}\left(\omega_{0}\right)$.

Proof. We have to show: $\varphi_{i} \omega \rightarrow \omega_{1}$ implies the existence of a $\psi \in \mathcal{G}^{r+1}\left(\omega_{0}\right)$ such that $\omega_{1}=\psi \omega$. We endow $P$ with the corresponding Kaluza-Klein metrics. Let $U_{x_{0}}$ be a normal chart centred at $x_{0} \in M$ of radius

$$
0<\varepsilon<\min \left\{r_{\mathrm{inj}}(g), r_{\mathrm{inj}}\left(g_{\omega}\right), r_{\mathrm{inj}}\left(g_{\omega_{1}}\right)\right\}
$$

and for $p_{0} \in \pi^{-1}\left(x_{0}\right)$

$$
\left\{h_{1}^{\omega}, \ldots, h_{n}^{\omega}, A_{1}^{\sharp}\left(p_{0}\right), \ldots, A_{m}^{\sharp}\left(p_{0}\right)\right\}
$$


be an orthonormal basis. We choose a subsequence $\left\{\varphi_{1 i}\right\}_{i}$ such that

(i) $\varphi_{1 i}^{-1}\left(p_{0}\right) \rightarrow q_{0} \in \pi^{-1}\left(x_{0}\right), q_{0}=p_{0} g_{0}, g_{0} \in G$;

(ii) $d \varphi_{1 i}^{-1}\left(h_{\nu}^{\omega}\right) \rightarrow \chi_{\nu} \in T_{q_{0}} P$.

Then $d \varphi_{i}^{-1}\left(A_{s}^{\sharp}\left(p_{0}\right)\right) \rightarrow A_{s}^{\sharp}\left(q_{0}\right)$. Denote by $e(\omega)$ the exponential map of $g_{\omega}$,

$$
B_{p_{0}}^{\varepsilon}:=\left\{t \in T_{p_{0}} P \mid g_{\omega}(t, t)<\varepsilon^{2}\right\} .
$$

We want to define $\psi$ on $e(\omega) B_{p_{0}}^{\varepsilon}$. Let $q=e(\omega) t$,

$$
t=\sum_{\nu=1}^{n} a^{\nu} h_{\nu}^{\omega}+\sum_{s=1}^{m} b^{s} A_{s}^{\sharp}\left(p_{0}\right)
$$

and set

$$
\psi^{-1}(q) \equiv \psi^{-1}(e(\omega) t):=e\left(\omega_{1}\right)\left[\sum_{\nu=1}^{n} a^{\nu} \chi^{\nu}+\sum_{s=1}^{m} b^{s} A_{s}^{\sharp}\left(p_{0}\right)\right]
$$

Proposition 4.3. If $t, t_{1} \in B_{p_{0}}^{\varepsilon}, e(\omega) t=\left[e(\omega) t_{1}\right] \cdot g$, then

$$
\psi^{-1}(e(\omega) t)=\psi^{-1}\left(e(\omega) t_{1}\right) \cdot g
$$

i.e. $\psi$ is a gauge transformation on $e(\omega) B_{p_{0}}^{\varepsilon}$. Equivariant continuation provides $\psi$ over $U_{x_{0}}$.

Proof. The proof follows from the following facts:

(i) $g_{\varphi \omega}=\varphi^{*} g_{\omega}$

(ii) $\varphi \circ e(\omega)=e(\varphi \omega) \circ d \varphi$

(iii) $e\left(\varphi_{1 i} \omega\right) \rightarrow e\left(\omega_{1}\right)$ uniform on compact subsets of $T_{p} P_{\varepsilon}=\left\{t \in T_{p} P \mid g_{\omega_{1}}(t, t) \leq \varepsilon\right\}$.

(iv) $\pi[e(\omega) t]=\pi\left[\psi^{-1} e(\omega) t\right]$.

(i), (ii), (iv) are easy calculations. For (iii) one uses $g_{\varphi_{i} \omega} \rightarrow g_{\omega_{1}}$ in $\mathcal{M}_{G}^{r}\left(\omega_{0}\right)=$ certain Sobolev space of metrics (cf. [6]) if $\varphi_{i} \omega \rightarrow \omega_{1}$ in $\mathcal{C}^{r}\left(\omega_{0}\right)$ and the boundedness of Christoffel symbols up to a certain order (cf. [7]).

Corollary 4.4. $\varphi_{1 i}^{-1} \rightarrow \psi^{-1}$ over $U_{x_{0}}$ in $C^{1}$.

Let $\eta=\omega-\omega_{1}$.

Corollary 4.5. $\nabla^{\omega} \widehat{\psi}=\eta$ over $U_{x_{0}}$.

Theorem 4.2 follows by a compact exhaustion $K_{1} \subseteq K_{2} \subseteq \cdots$ of M, finite cover of each $K_{i}$ by normal charts, the construction above and compatibility.

The next step is to show that the orbits are submanifolds of $\mathcal{C}^{r}\left(\omega_{0}\right)$. We recall the key

Theorem 4.6. Let $G$ be a Lie group, $X$ an analytic manifold, $(g, x) \mapsto g x$ an analytic left action. Assume that for $x \in X$ the orbit map $\rho(x): G \rightarrow X, g \mapsto g x$ is a subimmersion. Denote by $G_{x}$ the isotropy group of $x$.

(i) $G_{x}$ is a Lie subgroup and $T_{e}\left(G_{x}\right)=\operatorname{ker}\left(T_{e} \rho(x)\right)$.

(ii) The canonical map $i_{x}: G / G_{x} \rightarrow X,[g] \mapsto g x$ is an immersion with image $G \cdot x$.

(iii) If the orbit $G \cdot x$ is locally closed and $G$ satisfies second countability then $G \cdot x$ is a submanifold of $X$ and $i_{x}$ is an isomorphism from $G / G_{x}$ onto $G \cdot x$ and $T_{x}(G \cdot x)=$ $\operatorname{im}\left(T_{e} \rho(x)\right)$. 
R e mark. The smooth version of Theorem 4.6 is also valid.

Denote for $\omega \in \mathcal{C}^{r}\left(\omega_{0}\right)$ by $S(\omega)$ the symmetry group,

$$
S(\omega)=\left\{\varphi \in \mathcal{G}^{r+1}\left(\omega_{0}\right) \mid \varphi \omega=\omega\right\} .
$$

Proposition 4.7. Let $\varphi \in S(\omega)$. The following conditions are equivalent:

(i) $\widehat{\varphi} \exp \widehat{X} \in S(\omega)$

(ii) $\exp \widehat{X} \in S(\omega)$

(iii) $\nabla^{\omega} \tilde{X}=0$.

We omit the easy computational proof.

Corollary 4.8. $S(\omega)$ is discrete in $\mathcal{G}^{r+1}\left(\omega_{0}\right)$.

Proof. (iii) implies $(\widetilde{X}, \widetilde{X})_{x}=$ const. $\left(M^{n}, g\right)$ with $(I)$ and $\left(B_{k}\right) \operatorname{imply} \operatorname{vol}\left(M^{n}, g\right)=$ $\infty$, hence $\widetilde{X} \notin L^{2}$ and $\varphi$ is isolated.

COROLlary 4.9. $\mathcal{G}^{r+1}\left(\omega_{0}\right) / S(\omega)$ is an analytic manifold and

$$
\mathcal{G}^{r+1}\left(\omega_{0}\right) \stackrel{\pi}{\longrightarrow} \mathcal{G}^{r+1}\left(\omega_{0}\right) / S(\omega)
$$

a submersion.

LEMma 4.10. The map

$$
i_{\omega}: \mathcal{G}^{r+1}\left(\omega_{0}\right) / S(\omega) \rightarrow \mathcal{C}^{r}\left(\omega_{0}\right),[\varphi] \mapsto \varphi \omega
$$

is smooth.

Proof. It is sufficient to show that $i_{\omega} \circ \pi$ is smooth. But this is nothing else than the restriction of the smooth action map

$$
\Phi: \mathcal{G}^{r+1}\left(\omega_{0}\right) \times \mathcal{C}^{r}\left(\omega_{0}\right) \longrightarrow \mathcal{C}^{r}\left(\omega_{0}\right)
$$

to the submanifold $\mathcal{G}^{r+1}\left(\omega_{0}\right) \times\{\omega\}$.

Denote by $\Delta_{0}^{\omega}=\left(\nabla^{\omega}\right) * \nabla^{\omega}$ the Laplace operator on 0 -forms with values in $\mathbf{g}_{P}$, by $\sigma_{e}\left(\Delta_{0}^{\omega}\right)$ its essential spectrum. This an invariant of $\mathcal{C}^{r}\left(\omega_{0}\right)$, i.e. $\sigma_{e}\left(\Delta_{0}^{\omega}\right)=\sigma_{e}\left(\Delta_{0}^{\omega_{0}}\right)$. If

$$
\inf \sigma_{e}\left(\Delta_{0}^{\omega_{0}} \mid\left(\operatorname{ker}\left(\Delta_{0}^{\omega_{0}}\right)^{\perp}\right)\right)>0
$$

then $\operatorname{im} \Delta_{0}^{\omega_{0}}, \operatorname{im} \Delta_{0}^{\omega}, \operatorname{im} \nabla^{\omega_{0}}$ and $\operatorname{im} \nabla^{\omega}$ are closed.

Proposition 4.11. Assume $k-1 \geq r>n / 2+2, \omega \in \mathcal{C}^{r}\left(\omega_{0}\right)$ and $(\mathrm{Sp})$. Then $i_{\omega}$ is an injective immersion.

Proof. Let $o$ be the class of $e \in \mathcal{G}^{r+1}\left(\omega_{0}\right)$ in $\mathcal{G}^{r+1}\left(\omega_{0}\right) / S(\omega)$. We have to show

(i) $\operatorname{ker} T_{o}=\{0\}$;

(ii) $\operatorname{im} T_{o} i_{\omega} \subset T_{\omega} \mathcal{C}^{r}\left(\omega_{0}\right)$ is closed and admits complement.

(i) is very simple. If we consider (ii), an easy calculation shows $\operatorname{im} T_{o} i_{\omega}=\operatorname{im} T_{e} \Phi_{\omega}$ and $T_{e} \Phi_{\omega}=-\nabla^{\omega}$. (Sp) implies im $\nabla^{\omega}$ closed. The existence of an $L_{2}$-complement is assured by 
Proposition 4.12. Assume $k-1 \geq r>n / 2+2$ and the spectral condition (Sp) and $\omega \in \mathcal{C}^{r}\left(\omega_{0}\right)$. Then

$$
\Omega^{1,2, r}\left(\mathbf{g}_{P}, \nabla^{\omega_{0}}\right)=\operatorname{im} \nabla_{r+1}^{\omega} \oplus \operatorname{ker}\left(\nabla^{\omega}\right)_{r}^{*},
$$

where both spaces on the right-hand side are closed and $L^{2}$-orthogonal.

The proof is rather long and nontrivial since $\omega$ is nonsmooth and one has to establish Hodge theory for elliptic differential operators with Sobolev coefficients. We refer to [8], [13].

To apply Theorem 4.6 (iii), we need second countability of $\mathcal{G}^{r+1}\left(\omega_{0}\right)$ which is in general not satisfied since $\mathcal{G}^{r+1}\left(\omega_{0}\right)$ can have uncountably many components. It is easy to see that each element of the centre of $G$ generates one component. Therefore we restrict in the sequel ourselves to the subgroup $\mathcal{G}_{\bullet}^{r+1}\left(\omega_{0}\right)$ which consists of the components of $\mathcal{G}^{r+1}\left(\omega_{0}\right)$ generated by the centre of $G$ if this centre is countable or which equals to the component of the identity if the centre is uncountable. In the case $G=S U(2)$ the centre consists of two elements, i.e. of two components, each of them satisfies second countability.

The key role in the whole structure of the configuration space is played by the slice theorem which we now start to discuss.

We want to construct an equivariant tubular neighbourhood for each orbit. For this we need a Riemannian metric, a normal bundle and an exponential map. The tangent bundle of $\mathcal{C}^{r}\left(\omega_{0}\right)$ is simply $\mathcal{C}^{r}\left(\omega_{0}\right) \times \Omega^{1,2, r}\left(\mathbf{g}_{P}, \nabla^{\omega_{0}}\right)$. We define a weak Riemannian metric

$$
\omega \mapsto(,)_{w, \omega}=(,)_{w}
$$

as follows: $(\omega, \xi),(\omega, \eta) \in\{\omega\} \times \Omega^{1,2, r}\left(\mathbf{g}_{P}, \nabla^{\omega_{0}}\right)$,

$$
((\omega, \xi),(\omega, \eta))_{w}:=(\xi, \eta)_{w}:=\int_{M}(\xi, \eta)_{x} d \operatorname{vol}(x)
$$

$(,)_{w}$ is $\mathcal{G}^{r+1}\left(\omega_{0}\right)$-invariant.

A strong metric $(,)_{s t}$ on $\mathcal{C}^{r}\left(\omega_{0}\right)$ is given by

$$
\omega \mapsto \sum_{i=0}^{r}\left(\left(\nabla^{\omega}\right)^{i} \cdot,\left(\nabla^{\omega}\right)^{i} \cdot\right)_{w} .
$$

$(,)_{s t}$ is $\mathcal{G}^{r+1}\left(\omega_{0}\right)$-invariant and smooth and therefore defines an exponential map. Weak metrics in general do not have an exponential map. But in our case, with respect to $(,)_{w}$

$$
\operatorname{Exp}_{\omega} \eta=\omega+\eta .
$$

This exponential map is equivariant with respect to $\mathcal{G}^{r+1}\left(\omega_{0}\right)$. Denote

$$
N_{\omega}=\left\{\eta \in T \mathcal{C}^{r}\left(\omega_{0}\right) \mid \eta \perp \mathcal{G}_{\bullet}^{r+1}\left(\omega_{0}\right) \cdot \omega \text { w.r.t. }(,)_{w}\right\} .
$$

LEMMA 4.13. $N_{\omega}$ is a smooth subbundle of $T \mathcal{C}^{r}\left(\omega_{0}\right) \mid \mathcal{G}_{\bullet}^{r+1}\left(\omega_{0}\right) \cdot \omega$.

Proposition 4.14. Set

$$
N_{\omega}^{\varepsilon}:=\left\{\eta \in N_{\omega} \mid(\eta, \eta)<\varepsilon^{2}\right\} .
$$

There exists an $\varepsilon>0$ such that

$$
E=\operatorname{Exp} \mid N_{\omega}^{\varepsilon} \longrightarrow \mathcal{C}^{r}\left(\omega_{0}\right)
$$


is a $\mathcal{G}_{\bullet}^{r+1}\left(\omega_{0}\right)$-equivariant diffeomorphism onto an open $\mathcal{G}_{\bullet}^{r+1}\left(\omega_{0}\right)$-invariant neighbourhood of $\mathcal{G}_{\bullet}^{r+1}\left(\omega_{0}\right) \cdot \omega$.

We refer to [13] for the proof.

Corollary 4.15. There exists a $\mathcal{G}_{\bullet}^{r+1}\left(\omega_{0}\right)$ equivariant map $\mathcal{T}: N_{\omega} \rightarrow \mathcal{C}^{r}\left(\omega_{0}\right)$ which maps $N_{\omega}$ diffeomorphic onto an open $\mathcal{G}_{\bullet}^{r+1}\left(\omega_{0}\right)$-invariant neighbourhood of $\mathcal{G}_{\bullet}^{r+1}\left(\omega_{0}\right) \cdot \omega$.

Proof. Choose $\varepsilon$ as above, define $F: N_{\omega} \rightarrow N_{\omega}^{\varepsilon}$ as

$$
F(\eta):=\varepsilon \cdot \frac{\eta}{\sqrt{(\eta, \eta)_{s t}+1}}
$$

and set $\mathcal{T}=\operatorname{Exp} \circ F$.

$\mathcal{T}\left(N_{\omega}\right)$ is an open $\mathcal{G}_{\bullet}^{r+1}\left(\omega_{0}\right)$-invariant tubular neighbourhood of $\mathcal{G}_{\bullet}^{r+1}\left(\omega_{0}\right) \cdot \omega$ with bundle projection $\bar{\pi} \circ \mathcal{T}^{-1}$ onto $\mathcal{G}_{\bullet}^{r+1}\left(\omega_{0}\right) \cdot \omega$. The fibre $\mathcal{S}_{\omega_{1}}$ over $\omega_{1} \in \mathcal{G}_{\bullet}^{r+1}\left(\omega_{0}\right) \cdot \omega$ is called a slice. Then

$$
\mathcal{S}_{\omega_{1}}=\mathcal{T}\left(T_{\omega_{1}} \mathcal{C}^{r}\left(\omega_{0}\right) \cap N_{\omega}\right)=\operatorname{Exp}\left(T_{\omega_{1}} \mathcal{C}^{r}\left(\omega_{0}\right) \cap N_{\omega}^{\varepsilon}\right) \subset \mathcal{C}^{r}\left(\omega_{0}\right)
$$

Corollary 4.16. Let $\omega_{1} \in \mathcal{G}_{\bullet}^{r+1}\left(\omega_{0}\right) \cdot \omega$. Then

(i) $\varphi \in S\left(\omega_{1}\right)$ implies $\varphi \mathcal{S}_{\omega_{1}}=\mathcal{S}_{\omega_{1}}$;

(ii) $\varphi \in \mathcal{G}_{\bullet}^{r+1}\left(\omega_{0}\right), \omega_{2} \in \mathcal{S}_{\omega_{1}}$ and $\varphi \omega_{2} \in \mathcal{S}_{\omega_{1}}$ imply $\varphi \in S\left(\omega_{1}\right)$.

The corollary can be obtained by easy calculations.

(i) means that $S\left(\omega_{1}\right)$ acts on $\mathcal{S}_{\omega_{1}}$. (ii) shows that orbits different from the orbit $\mathcal{G}^{r+1}\left(\omega_{0}\right) \cdot \omega$ can intersect the slice several times and in this case

$$
\left(\mathcal{G}_{\bullet}^{r+1}\left(\omega_{0}\right) \cdot \omega_{2}\right) \cap \mathcal{S}_{\omega_{1}}=S\left(\omega_{1}\right) \cdot \omega_{2}
$$

We formulate now the main theorem of this section.

Theorem 4.17. Assume $\left(M^{n}, g\right)$ open with $(I)$ and $\left(B_{k}\right), k-1 \geq r>n / 2+2$, $\omega \in \mathcal{C}^{r}\left(\omega_{0}\right)$ and $\inf \sigma_{e}\left(\Delta_{0}^{\omega} \mid\left(\operatorname{ker} \Delta_{0}^{\omega}\right)^{\perp}\right)>0$. Let $U$ an open invariant neighbourhood of $\mathcal{G}_{\bullet}^{r+1}\left(\omega_{0}\right) \cdot \omega$. Then there exists a tubular neighbourhood of $\mathcal{G}_{\bullet}^{r+1}\left(\omega_{0}\right) \cdot \omega$ such that the complement of the closure of the tubular neighbourhood in $U$ is nonempty, i.e. there exists an equivariant map $\mathcal{T}: N_{\omega} \rightarrow U$ such that

(i) $\mathcal{T}\left(N_{\omega}\right)$ is open.

(ii) $\mathcal{T}: N_{\omega} \rightarrow \mathcal{T}\left(N_{\omega}\right)$ is a diffeomorphism.

(iii) $\overline{\mathcal{T}\left(N_{\omega}\right)} \subset U$.

(iv) $U-\overline{\mathcal{T}\left(N_{\omega}\right)} \neq \emptyset$.

Proof. Choose $\varepsilon_{1}$ as in Proposition 4.14. Then $\operatorname{Exp}\left(N_{\omega}^{\varepsilon_{1}}\right) \cap U$ is an open invariant neighbourhood of $\mathcal{G}_{\bullet}^{r+1}\left(\omega_{0}\right) \cdot \omega$. Choose $0<\varepsilon_{2}<\varepsilon_{1}$ such that

$$
\operatorname{Exp}\left(T_{\omega} \mathcal{C}^{r}\left(\omega_{0}\right) \cap N_{\omega}^{\varepsilon_{2}}\right) \subset U .
$$

$(,)_{s t}$ is invariant and Exp equivariant. Hence

$$
\mathcal{G}_{\bullet}^{r+1}\left(\omega_{0}\right) \cdot \operatorname{Exp}\left(T_{\omega} \mathcal{C}^{r}\left(\omega_{0}\right) \cap N_{\omega}^{\varepsilon_{2}}\right)=\operatorname{Exp}\left(N_{\omega}^{\varepsilon_{2}}\right)
$$

and $\operatorname{Exp}\left(N_{\omega}^{\varepsilon_{2}}\right) \subset U$. Choose $\varepsilon=\varepsilon_{2} / 2$ and apply Corollary 4.15. 
Corollary 4.18. The configuration space

$$
\mathcal{R}_{\bullet}^{r}\left(\omega_{0}\right):=\mathcal{C}^{r}\left(\omega_{0}\right) / \mathcal{G}_{\bullet}^{r+1}\left(\omega_{0}\right)
$$

is a regular topological space.

R e m a r k. The steps establishing Theorem 4.17 have been strongly modelled by Kondracki/Rogulski in [14].

5. The stratification of the configuration space. We will prove that the configuration space is a stratified space, the strata labelled by conjugacy classes of symmetry groups. In distinction from the compact case, in the open case uncountably many strata are possible. The procedure is once again modelled by [14].

Let $S$ be a (discrete) subgroup of $\mathcal{G}^{r+1}\left(\omega_{0}\right)$ such that there exists $\omega \in \mathcal{C}^{r}\left(\omega_{0}\right)$ with $S=$ $S(\omega)$. Then any conjugate subgroup is also symmetry group of a connection: $\varphi S \varphi^{-1}=$ $S(\varphi \omega)$. Denote by $(S)$ the conjugacy class of $S$ in $\mathcal{G}^{r+1}\left(\omega_{0}\right)$ where we admit conjugacy only by means of elements of $\mathcal{G}_{\bullet}^{r+1}\left(\omega_{0}\right)$. Denote

$$
\begin{aligned}
\mathcal{J} & :=\left\{(S) \mid S=S(\omega) \text { for some } \omega \in \mathcal{C}^{r}\left(\omega_{0}\right)\right\} \\
\mathcal{C}_{(S)}^{r}\left(\omega_{0}\right) & :=\left\{\omega \in \mathcal{C}^{r}\left(\omega_{0}\right) \mid S(\omega) \in(S)\right\} \\
\mathcal{C}_{S}^{r}\left(\omega_{0}\right) & :=\left\{\omega \in \mathcal{C}^{r}\left(\omega_{0}\right) \mid S(\omega)=S\right\} \\
\widetilde{\mathcal{C}}_{S}^{r}\left(\omega_{0}\right) & :=\left\{\omega \in \mathcal{C}^{r}\left(\omega_{0}\right) \mid S(\omega) \supset S\right\} .
\end{aligned}
$$

Their properties and mutual relations are described by the following propositions.

Proposition 5.1. For any $(S) \in \mathcal{J}, \mathcal{C}_{(S)}^{r}\left(\omega_{0}\right)$ is a smooth submanifold of $\mathcal{C}^{r}\left(\omega_{0}\right)$.

Proposition 5.2. $\widetilde{\mathcal{C}}_{S}^{r}\left(\omega_{0}\right)$ is an affine, closed subspace of $\mathcal{C}^{r}\left(\omega_{0}\right)$ and $\mathcal{C}_{S}^{r}\left(\omega_{0}\right)$ is an open subset of $\widetilde{\mathcal{C}}_{S}^{r}\left(\omega_{0}\right)$.

The proofs of Propositions 5.1, 5.2 essentially use tubular neighbourhoods and slices.

THEOREM 5.3. Let $S \subset \mathcal{G}^{r+1}\left(\omega_{0}\right)$ be the symmetry group of a connection $\omega_{1} \in \mathcal{C}^{r}\left(\omega_{0}\right)$. Then $\mathcal{C}_{S}^{r}\left(\omega_{0}\right)$ is a generic subset of $\widetilde{\mathcal{C}}_{S}^{r}\left(\omega_{0}\right)$, i.e. $\mathcal{C}_{S}^{r}\left(\omega_{0}\right) \subset \widetilde{\mathcal{C}}_{S}^{r}\left(\omega_{0}\right)$ open and dense.

We refer to [13] for the proof.

Corollary 5.4. $\mathcal{C}_{(S)}^{r}\left(\omega_{0}\right)$ is dense in $\mathcal{G}_{\bullet}^{r+1}\left(\omega_{0}\right) \cdot \widetilde{\mathcal{C}}_{S}^{r}\left(\omega_{0}\right)$.

LEMMA 5.5. $\mathcal{C}_{(S)}^{r}\left(\omega_{0}\right)$ is open in $\mathcal{G}_{\bullet}^{r+1}\left(\omega_{0}\right) \cdot \widetilde{\mathcal{C}}_{S}^{r}\left(\omega_{0}\right)$.

LEMMA 5.6. $\mathcal{G}_{\bullet}^{r+1}\left(\omega_{0}\right) \cdot \widetilde{\mathcal{C}}_{S}^{r}\left(\omega_{0}\right)$ is a closed subset $\mathcal{C}^{r}\left(\omega_{0}\right)$.

We conclude from Corollary 5.4 and Lemmas 5.5-5.6

ThEOREM 5.7. $\mathcal{C}_{(S)}^{r}\left(\omega_{0}\right)$ is a generic subset of $\mathcal{G}_{\bullet}^{r+1}\left(\omega_{0}\right) \cdot \widetilde{\mathcal{C}}_{S}^{r}\left(\omega_{0}\right)$ and

$$
\overline{\mathcal{C}_{(S)}^{r}\left(\omega_{0}\right)}=\mathcal{G}_{\bullet}^{r+1}\left(\omega_{0}\right) \cdot \widetilde{\mathcal{C}}_{S}^{r}\left(\omega_{0}\right) .
$$

The $\mathcal{C}_{(S)}^{r}\left(\omega_{0}\right),(S) \in \mathcal{J}$ are the bricks of $=\mathcal{G}_{\bullet}^{r+1}\left(\omega_{0}\right) \cdot \widetilde{\mathcal{C}}_{S}^{r} E\left(\omega_{0}\right)$ as shown by the following

Lemma 5.8. Assume $\omega^{\prime} \in \mathcal{G}_{\bullet}^{r+1}\left(\omega_{0}\right) \cdot \widetilde{\mathcal{C}}_{S}^{r}\left(\omega_{0}\right)$ and let $S^{\prime}=S\left(\omega^{\prime}\right)$. Then

$$
\mathcal{C}_{\left(S^{\prime}\right)}^{r} \subset \mathcal{G}_{\bullet}^{r+1}\left(\omega_{0}\right) \cdot \widetilde{\mathcal{C}}_{S}^{r}\left(\omega_{0}\right)
$$


To formulate now main theorems, we recall the notion of stratification used here. Let $X$ be a topological space and $\mathcal{D}$ a finite or countable family of nonempty subsets of $X$. $\mathcal{D}$ is called a regular stratification if it satisfies the following conditions:

(i) $\bigcup \mathcal{D}=X$.

(ii) $\Omega, \Omega^{\prime} \in \mathcal{D}, \Omega \cap \Omega^{\prime} \neq \emptyset$ imply $\Omega=\Omega^{\prime}$.

(iii) $\Omega, \Omega^{\prime} \in \mathcal{D}, \bar{\Omega} \cap \Omega^{\prime} \neq \emptyset$ imply $\bar{\Omega} \supset \Omega^{\prime}$.

(iv) $\Omega, \Omega^{\prime} \in \mathcal{D}, \bar{\Omega} \cap \Omega^{\prime} \neq \emptyset$ imply $\overline{\Omega^{\prime}} \cap\left(\Omega \cup \Omega^{\prime}\right)=\Omega^{\prime}$.

Giving up the condition of countability, we obtain the notion of quasistratification.

THEOREM 5.9. Let $\left(M^{n}, g\right)$ be open with $(I)$ and $\left(B_{k}\right), k-1 \geq r>n / 2+2$ and $\inf \sigma_{e}\left(\Delta_{0}^{\omega_{0}} \mid\left(\operatorname{ker} \Delta_{0}^{\omega_{0}}\right)^{\perp}\right)>0$. Then

$$
\left\{\mathcal{C}_{(S)}^{r}\left(\omega_{0}\right) \mid(S) \in \mathcal{J}\right\}
$$

is a regular quasistratification of $\mathcal{C}^{r}\left(\omega_{0}\right)$.

Finally we turn to the configuration space $\mathcal{R}_{\bullet}^{r}\left(\omega_{0}\right)=\mathcal{C}^{r}\left(\omega_{0}\right) / \mathcal{G}_{\bullet}^{r+1}\left(\omega_{0}\right)$.

LEMMA 5.10. $\mathcal{R}_{\bullet}^{r}\left(\omega_{0}\right)$ is a connected, metrizable space satisfying second countability.

This follows immediately from the properties of $\mathcal{C}^{r}\left(\omega_{0}\right)$, of the projection $\widehat{\pi}: \mathcal{C}^{r}\left(\omega_{0}\right) \rightarrow$ $\mathcal{R}_{\bullet}^{r}\left(\omega_{0}\right)$ and the metrization theorem.

For an orbit in $\mathcal{R}_{\bullet}^{r}\left(\omega_{0}\right)$ the symmetry groups of different elements of the orbit are conjugate by elements of $\mathcal{G}_{\bullet}^{r+1}\left(\omega_{0}\right)$, as we have already seen. Hence there exists a well defined map

$$
\text { type }: \mathcal{R}_{\bullet}^{r}\left(\omega_{0}\right) \longrightarrow \mathcal{J} \text {. }
$$

Define for $(S) \in \mathcal{J}$

$$
\mathcal{R}_{(S)}^{r}\left(\omega_{0}\right):=\operatorname{type}^{-1}((S))
$$

Remark.

(i) $\mathcal{C}_{(S)}^{r}\left(\omega_{0}\right)=\widehat{\pi}^{-1}\left(\operatorname{type}^{-1}((S))\right)$;

(ii) $\mathcal{R}_{(S)}^{r}\left(\omega_{0}\right)=\widehat{\pi}\left(\mathcal{C}_{(S)}^{r}\left(\omega_{0}\right)\right)$.

THEOREM 5.11. $\mathcal{R}_{(S)}^{r}\left(\omega_{0}\right)$ can be endowed with a unique manifold structure such that

$$
\widehat{\pi}: \mathcal{C}_{(S)}^{r}\left(\omega_{0}\right) \longrightarrow \mathcal{R}_{(S)}^{r}\left(\omega_{0}\right)
$$

is a smooth submersion.

LEMMA 5.12. $\overline{\mathcal{R}_{(S)}^{r}\left(\omega_{0}\right)}=\widehat{\pi}\left(\mathcal{G}_{\bullet}^{r+1}\left(\omega_{0}\right) \cdot \widetilde{\mathcal{C}}_{S}^{r}\left(\omega_{0}\right)\right)$

We finish with our main

Theorem 5.13. Assume $\left(M^{n}, g\right)$ open with $(I)$ and $\left(B_{k}\right), k-1 \geq r>n / 2+2$ and $\inf \sigma_{e}\left(\Delta_{0}^{\omega_{0}} \mid\left(\operatorname{ker} \Delta_{0}^{\omega_{0}}\right)^{\perp}\right)>0$. Then

$$
\mathcal{D}=\left\{\mathcal{R}_{(S)}^{r}\left(\omega_{0}\right) \mid(S) \in \mathcal{J}\right\}
$$

is a regular quasistratification of the configuration space

$$
\mathcal{R}_{\bullet}^{r}\left(\omega_{0}\right)=\mathcal{C}^{r}\left(\omega_{0}\right) / \mathcal{G}_{\bullet}^{r+1}\left(\omega_{0}\right) .
$$


(i), (ii) are clear. (iii) and (iv) can be proved by the straight use of our definitions and constructions.

\section{References}

[1] N. Bourbaki, Éléments de mathématique, Fasc. XXXIII, Variétés différentielles et analytiques, Fascicule de résultates (Paragraphes 1 à 7), Hermann, Paris, 1971.

[2] N. Bourbaki, Éléments de mathématique, Fasc. XXXVIII, Groupes et algèbres de Lie, Hermann, Paris, 1972.

[3] J. Eichhorn, Elliptic differential operators on noncompact manifolds, in: Seminar Analysis of the Karl-Weierstrass-Institute of Mathematics (Berlin, 1986/87), Teubner-Texte Math. 106, Leipzig, 1988, 4-169.

[4] J. Eichhorn, Gauge theory on open manifolds of bounded geometry, Internat. J. Modern Phys. A 7 (1992), 3927-3977.

[5] J. Eichhorn, The manifold structure of maps between open manifolds, Ann. Global Anal. Geom. 11 (1993), 253-300.

[6] J. Eichhorn, Spaces of Riemannian metrics on open manifolds, Results Math. 27 (1995), 256-283.

[7] J. Eichhorn, The boundedness of connection coefficients and their derivatives, Math. Nachr. 152 (1991), 145-158.

[8] J. Eichhorn, Differential operators with Sobolev coefficients, in preparation.

[9] J. Eichhorn, The invariance of Sobolev spaces over noncompact manifolds, in: Symposium "Partial Differential Equations" (Holzhau, 1988), Teubner-Texte Math. 112, Leipzig, 1989, 73-107.

[10] J. Eichhorn and J. Fricke, The module structure theorem for Sobolev spaces on open manifolds, Math. Nachr. (to appear).

[11] J. Eichhorn and R. Schmid, Form preserving diffeomorphisms on open manifolds, Math. Nachr. (to appear).

[12] A. E. Fischer, The internal symmetry group of a connection on a principal fibre bundle with applications to gauge field theory, Comm. Math. Phys. 113 (1987), 231-262.

[13] G. Heber, Die Topologie des Konfigurationsraumes der Yang-Mills Theorie über offenen Mannigfaltigkeiten beschränkter Geometrie, Ph.D. thesis, Greifswald, 1994.

[14] W. Kondracki and J. Rogulski, On the stratification of the orbit space for the action of automorphisms on connections, Dissertationes Math. (Rozprawy Mat.) 250 (1986).

[15] W. Kondracki and P. Sadowski, Geometric structure on the orbit space of gauge connections, J. Geom. Phys. 3 (1986), 421-434. 